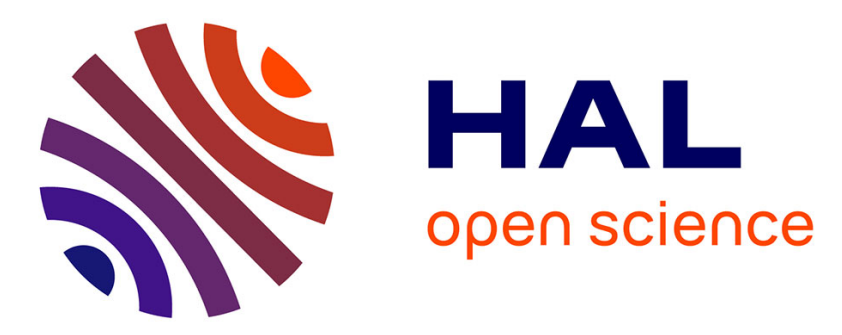

\title{
In vivo ultrasound imaging of the bone cortex
}

Guillaume Renaud, Pieter Kruizinga, Didier Cassereau, Pascal Laugier

\section{To cite this version:}

Guillaume Renaud, Pieter Kruizinga, Didier Cassereau, Pascal Laugier. In vivo ultrasound imaging of the bone cortex. Physics in Medicine and Biology, 2018, 63 (12), pp.125010. 10.1088/13616560/aac784 . hal-01971778

\section{HAL Id: hal-01971778 \\ https: / hal.sorbonne-universite.fr/hal-01971778}

Submitted on 7 Jan 2019

HAL is a multi-disciplinary open access archive for the deposit and dissemination of scientific research documents, whether they are published or not. The documents may come from teaching and research institutions in France or abroad, or from public or private research centers.
L'archive ouverte pluridisciplinaire HAL, est destinée au dépôt et à la diffusion de documents scientifiques de niveau recherche, publiés ou non, émanant des établissements d'enseignement et de recherche français ou étrangers, des laboratoires publics ou privés. 


\title{
In vivo ultrasound imaging of the bone cortex
}

\author{
Guillaume Renaud ${ }^{1} \odot$, Pieter Kruizinga ${ }^{2}$, Didier Cassereau ${ }^{1}$ and Pascal Laugier ${ }^{1}$ \\ 1 Sorbonne Université, UPMC, CNRS UMR 7371, INSERM UMR S 1146, Laboratoire d'Imagerie Biomédicale, Paris, France \\ 2 Dept. Biomedical Engineering, ThoraxCenter, Erasmus Medical Center, Rotterdam, Netherlands \\ E-mail:guillaume.renaud@upmc.fr
}

Keywords: ultrasound imaging, cortical bone, refraction, ray tracing, elastic anisotropy

Supplementary material for this article is available online

\begin{abstract}
Current clinical ultrasound scanners cannot be used to image the interior morphology of bones because these scanners fail to address the complicated physics involved for exact image reconstruction. Here, we show that if the physics is properly addressed, bone cortex can be imaged using a conventional transducer array and a programmable ultrasound scanner. We provide in vivo proof for this technique by scanning the radius and tibia of two healthy volunteers and comparing the thickness of the radius bone with high-resolution peripheral x-ray computed tomography. Our method assumes a medium that is composed of different homogeneous layers with unique elastic anisotropy and ultrasonic wave-speed values. The applicable values of these layers are found by optimizing image sharpness and intensity over a range of relevant values. In the algorithm of image reconstruction we take wave refraction between the layers into account using a ray-tracing technique. The estimated values of the ultrasonic wave-speed and anisotropy in cortical bone are in agreement with ex vivo studies reported in the literature. These parameters are of interest since they were proposed as biomarkers for cortical bone quality. In this paper we discuss the physics involved with ultrasound imaging of bone and provide an algorithm to successfully image the first segment of cortical bone.
\end{abstract}

\section{Introduction}

The powerful image of the bones inside the hand of Mr Röntgen's wife, in 1895, seen with what has later been called x-rays, has by all means been the catalyst that stimulated the field of medical imaging. Still today x-ray imaging remains a technique of choice for imaging bone disorders. Because of their high density (and hence high $\mathrm{x}$-ray attenuation), bones produce a great contrast in an $\mathrm{x}$-ray image with respect to soft tissues. Bone scintigraphy and magnetic resonance imaging (MRI) are also commonly used for diagnozing bone disorders. Ultrasound imaging is extensively used for the examination of soft tissues. Although ultrasound imaging was shown to accurately image the outer surface of bones and thus allowing for the diagnosis of suspected bone fractures (Beltrame et al 2012), current clinical ultrasound scanners fail to reveal the inner structure of bones. In this work, our objective is to reassess the feasibility of imaging bones with ultrasonography.

Ultrasound imaging relies on the transmission of a series of ultrasound beams, the recording of echoes reflected by the medium and an algorithm of image reconstruction (Shung 2015). An ultrasound probe contains an array of individual active elements that are able to generate and record ultrasound waves. The reconstruction of an ultrasound image is conventionally done by delaying the received echoes with respect to every image point and summing these echoes over all receive elements. This algorithm is called delay-and-sum and is the technique of choice for image reconstruction in most ultrasound scanners. The amount of delay applied to each echo is based on the calculation of the round-trip travel times of the ultrasound wave for all image points; from the transmitting elements of the probe to the image point (forward propagation), and from the image point to the receiving elements of the probe (backward propagation). Figure 1 illustrates the principle of a delay-and-sum algorithm. The recorded sample values evaluated at specific instants (the calculated round-trip travel times) are 


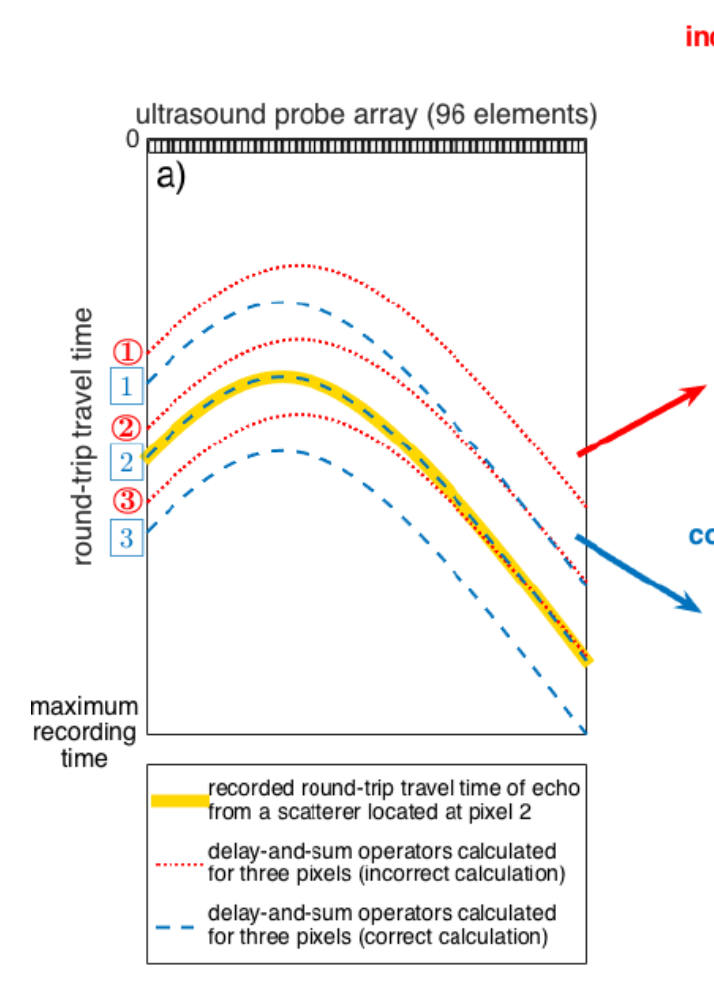

incorrect calculation of round-trip travel times

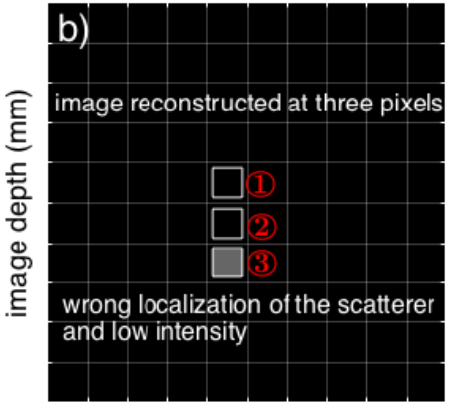

image width $(\mathrm{mm})$

correct calculation of round-trip travel times

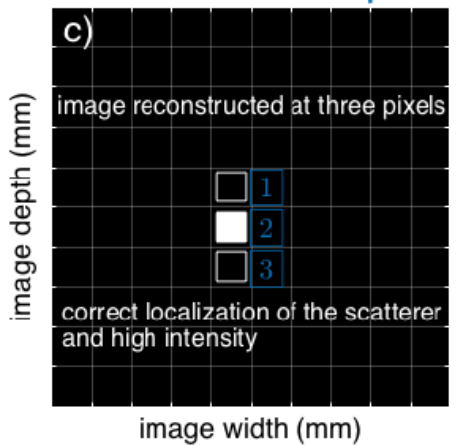

Figure 1. Principle of a delay-and-sum algorithm illustrated with the image reconstruction at three adjacent pixels. A scatterer (interface, small heterogeneity...) is located at pixel 2. If the calculation of the round-trip travel times is incorrect (panel (a)), the scatterer will appear at a wrong location and with a weak intensity (panel (b)). A correct calculation of the round-trip travel times (panel (a)) leads to a correct localization of the scatterer and a high intensity (panel (c)).

summed over the receiving elements of the probe, the sum gives a high image intensity if a scatterer is located at the image point or a low image intensity if not. In current clinical ultrasound scanners, the calculation of the round-trip travel times assumes a simplified medium with a speed of sound that is uniform and equal to the average value in soft tissues $\left(1540 \mathrm{~m} \mathrm{~s}^{-1}\right)$. A corollary is that an ultrasound ray is assumed to follow a straight path between the ultrasound probe and a point in the medium. This assumption is acceptable in soft tissues because the difference between the average value and the true speed of sound is at most $\pm 10 \%$. However this assumption does not hold for cortical bone that forms the cortex (outer shell) of bones. Indeed the speed of sound in cortical

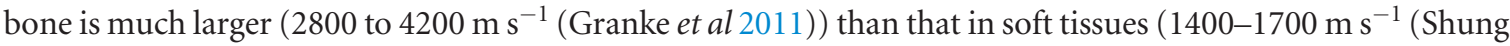
2015)), which leads to substantial refraction when an ultrasound wave traverses an interface between soft tissue and cortical bone. The physics of refraction tells us that the propagation direction of a wave changes when traversing an interface between two media with a different speed of sound. Therefore refraction corrupts the calculation of the round-trip travel times. If the calculation of the round-trip travel times is incorrect, the delayand-sum algorithm fails to reveal the existence and the true location of a scatterer (figures 1 (a) and (b). That is why the objects located behind (deeper than) the first interface between soft tissue and bone appear blurred, with a weak intensity and at a wrong spatial location, in an image reconstructed by current clinical scanners.

It is largely thought that the reasons why clinical ultrasound scanners fail to image bone are ((i) the large ultrasound attenuation in cortical bone (close to $10 \mathrm{~dB} / \mathrm{cm} / \mathrm{MHz}$ (Zheng et al 2007, Shung 2015)) and (ii) the low transmission through an interface between soft tissue and cortical bone caused by the large acoustic impedance mismatch. These two issues relate to technological limitations, and the performances of hardware electronics in ultrasound scanners and the sensitivity of ultrasound probes have tremendously improved in the past 20 years. Although these effects play their part, we demonstrate in this work that the main issue is refraction, that must be considered for the correct calculation of the round-trip travel times.

Prior attempts to image bone with ultrasound were limited to ex vivo experiments or in silico studies (Lasaygues et al 2005, 2006, Li et al 2013, Zheng et al 2015, Bernard et al 2017), moreover the ultrasonic wavespeed in cortical bone was considered to be known and the anisotropy of the ultrasonic wave-speed was ignored. In this work, we demonstrate that ultrasound imaging of the bone cortex is possible in vivo and that the measurement of the cortical thickness at the radius of two healthy volunteers is accurate. This is of clinical interest since cortical thickness at the radius measured with high-resolution peripheral x-ray computed tomography was shown to improve fracture prediction (Chevalley et al 2013). Moreover our method outputs estimates of the 
ultrasonic wave-speed and its anisotropy in cortical bone, which was proposed as a biomarker of the quality of cortical bone tissue since ultrasonic wave-speed is determined by tissue elasticity and mass density (Granke et al 2011).

The algorithm developed for reconstructing an ultrasound image of the first segment of the bone cortex, that is to say the cortical bone layer closest to the ultrasound probe, is introduced in section 2. Section 3 describes the methodology for estimating the input parameters required by the algorithm for image reconstruction. Ultrasound images of the bone cortex obtained with a conventional clinical probe at the radius and tibia of two healthy volunteers are shown in section 4 , and we show that the cortical thickness at the radius measured in the ultrasound image agrees with that measured with high-resolution peripheral x-ray computed tomography. In section 5 we discuss four points: (i) the choice of the type of transmit ultrasound beam, (ii) why our method differs from previous in vivo approaches aiming to characterize cortical bone with ultrasound, (iii) the added value of considering the elastic anisotropy of cortical bone for image reconstruction and (iv) the possibility of imaging the entire cross-section of a long bone with ultrasonography.

\section{Ultrasound image reconstruction of the first segment of cortical bone}

We developed an algorithm for reconstructing an ultrasound image of the first segment of the bone cortex, that is to say the cortical bone layer closest to the ultrasound probe. For comparison, the images were also reconstructed with the conventional approach. Both methods are based on a delay-and-sum algorithm. The sole difference between the two approaches is the calculation of the round-trip travel times. In conventional image reconstruction, the wave-speed in the medium is assumed uniform and equals the average value for soft tissues $\left(1540 \mathrm{~m} \mathrm{~s}^{-1}\right)$. Therefore conventional image reconstruction hypothesizes that ultrasound waves follow straight paths. Panels (a) and (b) in figure 2 illustrate the configuration considered in conventional image reconstruction, for transverse and longitudinal images of a bone. Our approach considers a medium that is composed of different homogeneous layers (silicone front layer of the ultrasound probe, cutaneous tissue and cortical bone), therefore wave refraction is taken into account. As a result, an ultrasonic ray does not follow straight paths (figure 2, panels (c) and (d)). Moreover the anisotropy of the elasticity of cortical bone is considered, this implies that the wave-speed in cortical bone depends on the direction of propagation. Seismologists have extensive experience in producing images of the structure of the Earth based on the analysis of elastic waves which follow the same laws of physics as ultrasound waves. The subsurface of the Earth contains layers of solid materials (often with anisotropic elasticity) and liquids, consequently it is very similar to a region of the human body containing bone and soft tissues. Interestingly our approach resembles Kirchhoff migration developed by seismologists for imaging the subsurface of the Earth (Etgen et al 2009). We provide the complete $\mathrm{C}++$ code for image reconstruction of bone in the online supplementary material available at stacks.iop.org/PMB/63/125010/mmedia.

\subsection{Delay-and-sum algorithm}

The first step of a delay-and-sum algorithm is the calculation of the round-trip travel times for each point $P$ of the image, for each transmit beam, and for each receive elements of the probe array. As shown in figure 2, the round-trip travel time is the sum of a transmit travel time $t_{T}(i, P)$ (from the transmit element $i$ to image point $P$ ) and a return travel time $t_{R}(j, P)$ (from image point $P$ to the receive element $j$ ). The calculation of the round-trip travel times is only possible if the geometry of the interfaces between the layers and the wave-speed in each of the three layers are known. Obviously this information is unknown in cutaneous tissue and cortical bone, we developed a method to estimate the required parameters in vivo and describe it in section 3. The wave-speed and the thickness of the silicone front layer of the ultrasound probe were determined in a separate experiment, we found $930 \mathrm{~m} \mathrm{~s}^{-1}$ and $1.4 \mathrm{~mm}$ respectively. In our algorithm for image reconstruction, the calculation of the travel time from an element of the probe array to an image point was performed with a bending ray tracing technique which is extensively used by seismologists (Waltham et al 1998). In this method, the law of refraction (Snell's law) is imposed at all interfaces but one, and the angle of the emerging ray at the element is varied until a minimum travel time is obtained (Fermat's principle). In conventional image reconstruction, the calculation of the roundtrip travel times reduces to a simple geometry problem since an ultrasound ray from an element of the probe array to an image point is straight.

The second step of a delay-and-sum algorithm is a summation of the recorded signals along traces described by the calculated round-trip travel times (see figure 1), for each image point, over all transmissions and over all receive elements. The amplitude in the image $I(P)$ at point $P$ is given by: $I(P)=\sum_{i=1}^{M} \sum_{j=1}^{N} W(P, i, j) \times D\left[t=t_{T}(i, P)+t_{R}(j, P), i, j\right] . M$ and $N$ are the number of transmitting elements and receiving elements, respectively. $D\left[t=t_{T}(i, P)+t_{R}(j, P), i, j\right]$ is the received data value when element $i$ transmits and element $j$ records, evaluated at the time $t=t_{T}(i, P)+t_{R}(j, P)$ and weighted by an appropriate factor $W(P, i, j)$. We adopted a standard weighting function (as discussed in Nikolov et al (2010)): 


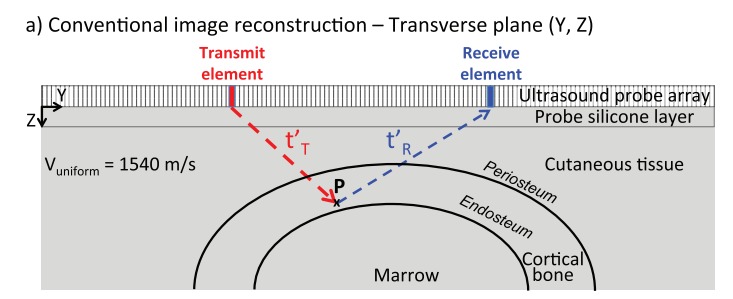

c) Image reconstruction in this work - Transverse plane $(Y, Z)$

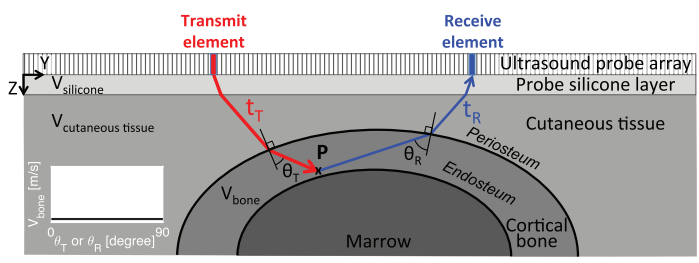

b) Conventional image reconstruction - Longitudinal plane $(X, Z)$ Transmit Receive

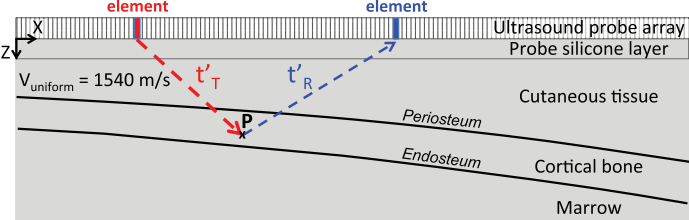

d) Image reconstruction in this work - Longitudinal plane $(X, Z)$

d) Image reconstruction in this work - Longituding
Transmit

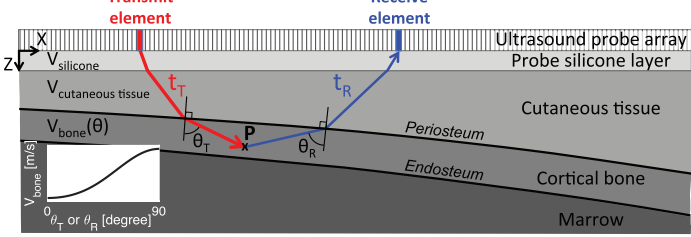

Figure 2. Schematics illustrating the simplified physics assumed in conventional image reconstruction (panels (a) and (b)) and the physics addressed in this work (panels $(\mathrm{c})$ and $(\mathrm{d})$ ) for reconstructing an image of the first segment of cortical bone. In conventional image reconstruction, the medium is considered uniform with a unique wave-speed value $\left(1540 \mathrm{~m} \mathrm{~s}^{-1}\right)$, hence wave refraction is neglected. In this work, we consider a medium with three homogeneous layers: the silicone front layer of the ultrasound probe, cutaneous tissue and cortical bone. Unlike in a transverse plane [Y,Z] (panel (c)), in a longitudinal plane [X, Z] of a long bone (panel (d)), ultrasound wave-speed in cortical bone depends on the direction of propagation (see insert in panel (d)). A delay-andsum algorithm for image reconstruction requires the calculation of the round-trip travel time (for each image point $P$ ), it is the sum of a transmit travel time $t_{T}\left(t_{T}^{\prime}\right.$ for conventional image reconstruction) and a return travel time $t_{R}\left(t_{R}^{\prime}\right.$ for conventional image reconstruction). Obviously $t_{T} \neq t_{T}^{\prime}$ and $t_{R} \neq t_{R}^{\prime}$, this explains why conventional image reconstruction fails to image bone.

$W(P, i, j)=1$ if the emerging angles from the transmit and receive elements $i$ and $j$ to point $P$ are smaller than an acceptance angle (Nikolov et al 2010$)$, otherwise $W(P, i, j)=0$.

Note that we did not reconstruct the complete cross-section image of the bone (see figure 2), the images shown in section 4 were reconstructed until the first inner surface of bone (endosteum) because of the existence of strong multiple reflections. This issue is further discussed in section 5.

\subsection{Anisotropy of the ultrasonic wave-speed in cortical bone}

Cortical bone is a solid material, therefore two types of ultrasonic waves exist: compressional and shear waves. However our approach disregards any event recorded after the arrival time of the compressional wave reflected by the interface between cortical bone and marrow (endosteum), including mode-converted shear waves since the wave-speed of a compressional wave in cortical bone is about twice as high as that of a shear wave (Granke et al 2011). Therefore only compressional waves will be considered here. The calculation of the round-trip travel times for image points located in cortical bone needs to take into account the dependence of the wavespeed on the direction of propagation. Indeed ex vivo studies have shown that the elasticity of human cortical bone is anisotropic (Granke et al 2011, Bernard et al 2016). Elastic anisotropy of cortical bone measured at the millimeter-scale has two origins: the oriented cortical porosity (Haversian canals) and micrometer-scale structure of the mineralized collagen matrix. The physics of the propagation of elastic waves in a medium with anisotropic elasticity is rather complicated and will not be detailed in this paper. The elasticity of cortical bone is well described with transverse isotropy (Granke et al 2011, Bernard et al 2016). A medium with transverse isotropy possesses a plane of isotropy in which the wave-speed does not depend on the propagation direction. For the central part of a long bone (diaphysis), the plane of isotropy is normal to the axis of the bone. In any plane that is not parallel to the plane of isotropy, the wave-speed depends on the propagation direction. The compressional wave-speed is about $20 \%$ higher in a direction parallel to the axis of a long bone than in a direction normal to the axis (Granke et al 2011). Therefore we used a model of weak elastic anisotropy proposed by seismologists (Thomsen 1986). The wave-speed of an ultrasound ray in an anisotropic medium is called the group velocity. The group velocity of the compressional wave in cortical bone $V_{\text {bone }}(\theta)$ will be modeled with the following equation:

$$
V_{\text {bone }}(\theta)=V_{\text {axial }}-\left(V_{\text {axial }}-V_{\text {radial }}\right) \times\left[\beta \sin ^{2}(\theta) \cos ^{2}(\theta)+\cos ^{4}(\theta)\right],
$$

$V_{\text {radial }}$ is the compressional wave-speed in the plane of isotropy. $V_{\text {axial }}$ is the compressional wave-speed in the direction of the axis of the long bone. $\beta$ is an anisotropy form parameter. $\theta$ is the angle between the ultrasound ray in cortical bone and the normal to the interface between soft tissue and bone. The inserts in panels (c) and (d) in figure 2 depict the dependence of the wave-speed in cortical bone on the angle $\theta$, as described by equation (1). 
a) Configuration for a transverse image

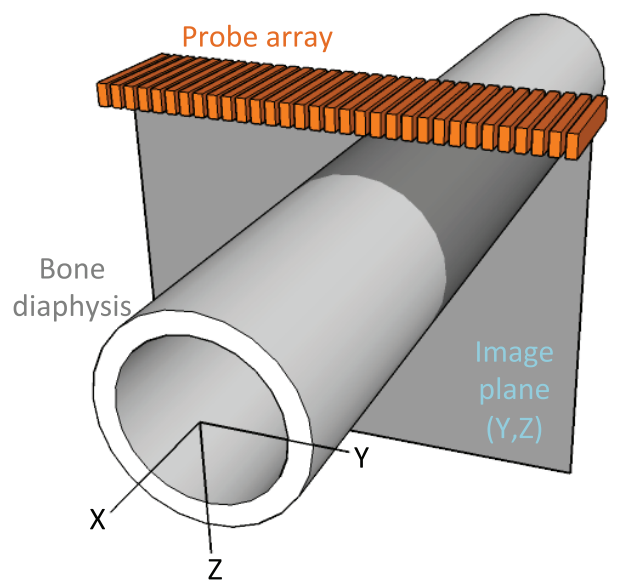

b) Configuration for a longitudinal image

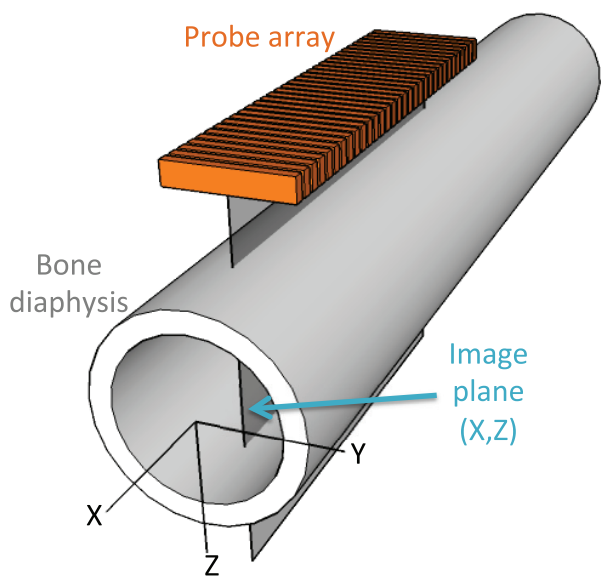

Figure 3. Graphical overview of the two imaging scenarios. (a) Position of the probe for a transverse image, in this configuration the image plane $(\mathrm{Y}, \mathrm{Z})$ corresponds to the plane of elastic isotropy of cortical bone. (b) Position of the probe for a longitudinal image, the elasticity of cortical bone in this image plane $(\mathrm{X}, \mathrm{Z})$ is anisotropic. Only the region that is examined by the ultrasound probe is depicted.

\section{Materials and methods}

In this work we consider transverse and longitudinal images of the diaphysis (central part) of long bones (figure 3). We provide here a brief overview of the methodology, details are given thereafter. The flowchart in figure 4 summarizes the methodology.

(1) Transverse view: The ultrasound probe was first positioned to obtain a transverse image (figure 3(a). The wave-speed in cutaneous tissue was obtained by searching for optimal focus quality (maximum intensity and sharpness of the image) of the reflection at the periosteum (outer surface of bone). To do so, the same ultrasound data was used to reconstruct a series of images of the cutaneous tissue layer, obtained with different values of the wave-speed in cutaneous tissue. Next, the periosteum was automatically segmented in the ultrasound image showing the highest focus quality using a technique based on Dijkstra's algorithm (Dijkstra 1959, Hong et al 2012). The segmentation algorithm seeks the shortest path that follows the interface with highest intensity in the ultrasound image, by maximizing a merit figure (here the sum of the pixel values along the path). Then the result of the segmentation was approximated by a parabola. In the transverse plane $(\mathrm{Y}, \mathrm{Z})$, the wave-speed in cortical bone is isotropic and named $V_{\text {radial }}$. The value of $V_{\text {radial }}$ was estimated by searching for optimal focus quality of the reflection at the endosteum (inner surface of bone). To do so, a series of images of the cortical bone layer was reconstructed with different values of $V_{\text {radial }}$. At this point, an accurate image in the transverse view can be reconstructed by splicing the optimal images of the cutaneous tissue layer and the cortical bone layer together.

(2) Longitudinal view: In a second step, the ultrasound probe was rotated 90 degrees, to allow a longitudinal image of the diaphysis (figure 3(b). As with the transverse image, the wave-speed in cutaneous tissue was estimated, the periosteum was automatically segmented and approximated by a parabola. In the longitudinal plane $(\mathrm{X}, \mathrm{Z})$, the wave-speed in cortical bone is anisotropic. The reconstruction of a longitudinal image of the cortical bone layer requires the use of equation (1) to describe the dependence of the wave-speed in cortical bone on the propagation direction. This model has three constants: $V_{\text {radial }}, V_{\text {axial }}$ and $\beta$. Instead of solving a three-dimensional optimization problem to find these three constants, we propose to use the value of $V_{\text {radial }}$ estimated in the transverse view and a specific signal processing to measure $V_{\text {axial }}$ (measurement of the head-wave velocity). At last, the anisotropy form parameter in cortical bone $\beta$ was estimated by searching for optimal focus quality of the reflection at the endosteum in the longitudinal image of the cortical bone layer. To do so, a series of images of the cortical bone layer was reconstructed with different values of $\beta$. As with the transverse image, an accurate image in the longitudinal view could be reconstructed by splicing the optimal images of the cutaneous tissue layer and the cortical bone layer together. 


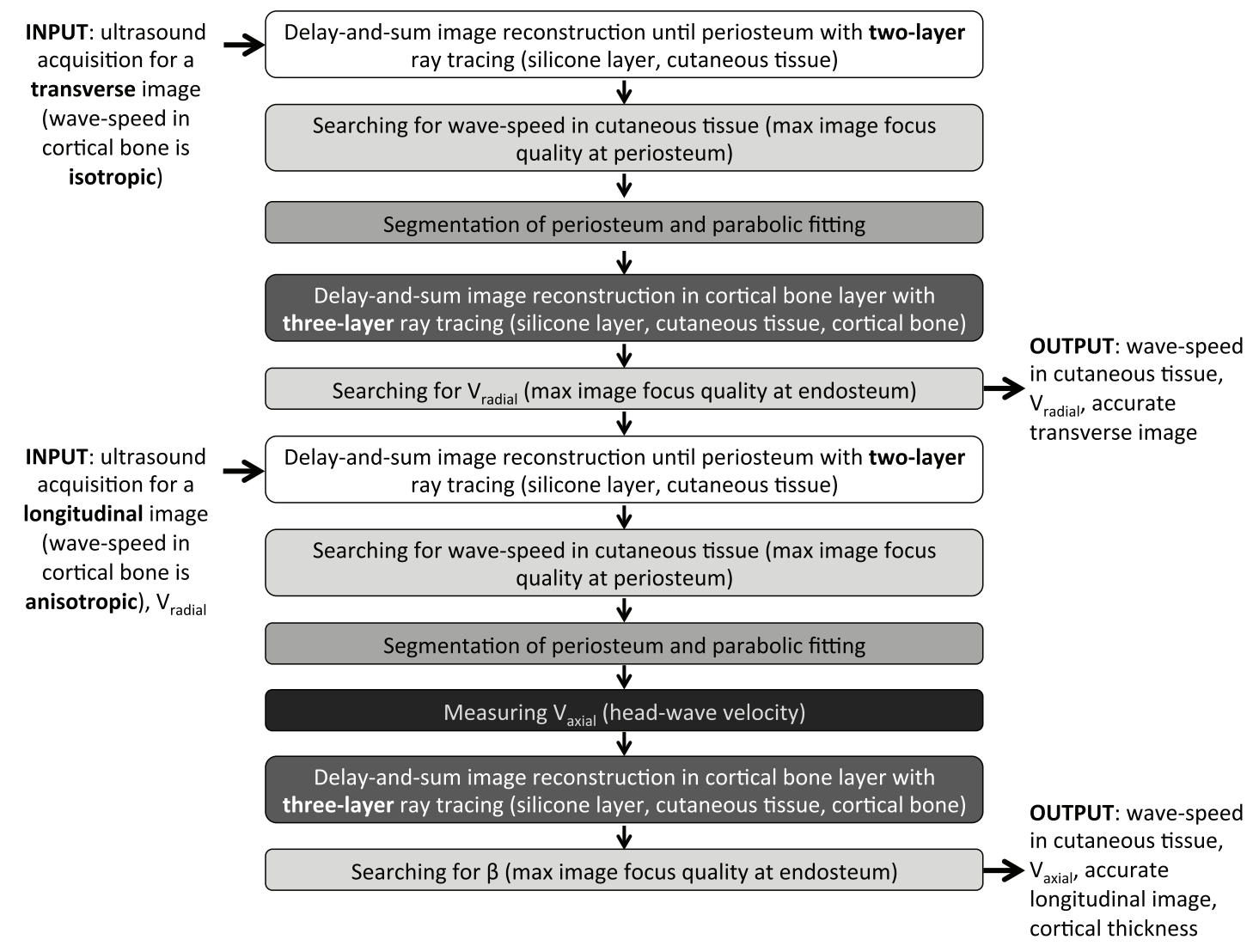

Figure 4. Graphical overview of the methodology. Steps applying the same processing are displayed with the same shade of gray.

\subsection{Ultrasound acquisitions}

For the ultrasound data acquisition we employed a synthetic aperture scheme (Jensen et al 2006), (also named full matrix capture (Holmes et al 2005)) in which every element in the array subsequently transmits a quasi-spherical diverging wavefront, followed by a full array capture of the received echo signals. These inter-element pulseecho signals were recorded using a fully programmable ultrasound system (Vantage, Verasonics Inc., Redmond, WA, USA) equipped with a $2.5 \mathrm{MHz}$ linear phased-array transducer (P4-1 ATL/Philips, Bothell, WA, USA; pitch $0.295 \mathrm{~mm}, 96$ elements), resulting in a total of $96 \times 96$ pulse-echo signals per acquisition.

Ultrasound recordings were acquired at the left radius and at the left tibia of two healthy volunteers recruited from the laboratory staff. An informed written consent was obtained from the volunteers. The radius and tibia were chosen because these two peripheral sites were largely studied in vivo for assessing the fracture risk with ultrasonic techniques or x-ray computed tomography (Chevalley et al 2013, Olszynski et al 2013). For the ultrasound examination at the radius, the center of the ultrasound probe was placed $80 \mathrm{~mm}$ away from the distal end of the radius (wrist joint). For the ultrasound examination at the tibia, the ultrasound probe was positioned at the mid-diaphysis (central part). For both radius and tibia examinations, the inter-element responses were acquired in two configurations (figure 3): (1) with the probe array positioned transverse to the axis of the bone and (2) with the probe array positioned parallel to the axis of the bone. Each measurement configuration was repeated five times with systematic repositioning of the probe. Real-time image reconstruction was not available during the acquisition, so the positioning of the probe was guided by displaying an image of the echoes received by the transmitting elements, i.e. as if each element was operated in pulse-echo mode. Using this image, the position of the probe was adjusted until maximum reflection at the periosteum and at the endosteum was observed. The periosteum and endosteum were identified due to their high echogenicity.

\subsection{Search for optimal focus quality in the ultrasound image and estimation of the wave-speed in cutaneous tissue and in cortical bone $\left(V_{\text {radial }}\right.$ and $\left.\beta\right)$}

The concept of optimal focus quality is not rigorously defined. It can be obtained by searching for maximum intensity and maximum sharpness of the image (Treeby et al 2011). The intensity of the ultrasound image was calculated as the sum of the squared amplitude of the image envelope. The sharpness of the ultrasound image was assessed by calculating the normalized variance of the image envelope (Treeby et al 2011). We have used these two metrics to estimate three physical parameters: wave-speed in cutaneous tissue, $V_{\text {radial }}$ and $\beta$. 

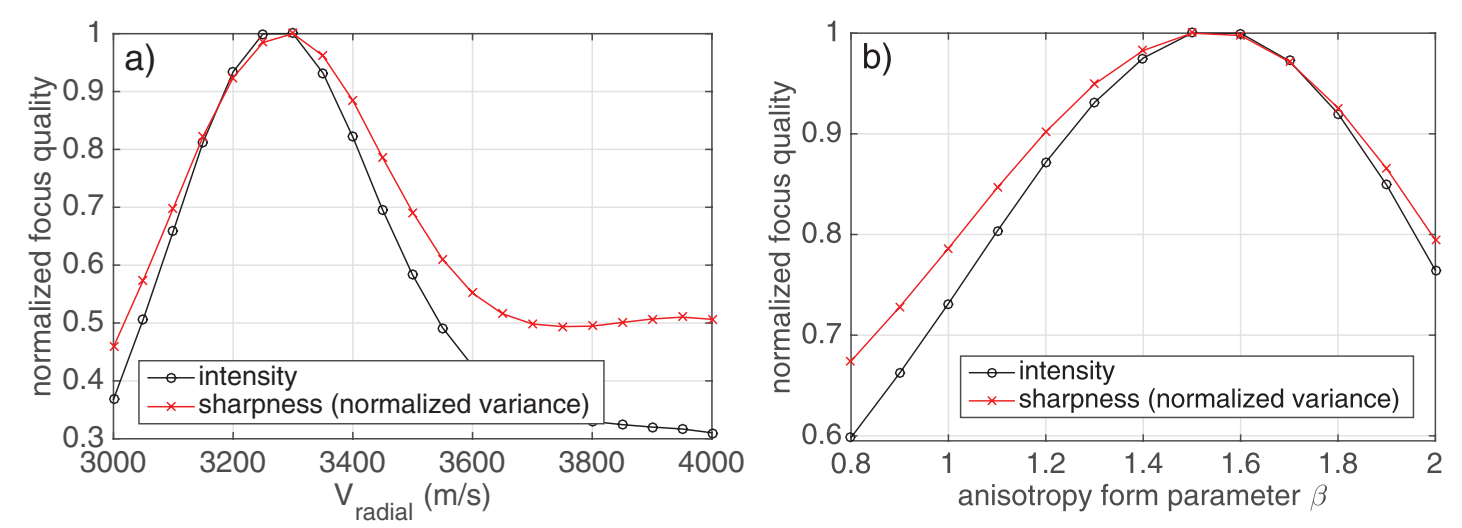

Figure 5. Exemplary variations of the two metrics of image focus quality obtained as a function of the radial wave-speed in cortical bone $V_{\text {radial }}$ (panel (a)) and the anisotropy form parameter in cortical bone $\beta$ (panel (b)). The metrics of intensity and sharpness show a maximum corresponding to the optimal value for the radial wave-speed and the anisotropy form parameter.

The wave-speed in cutaneous tissue was estimated by searching, within a relevant range (1400-1700 $\left.\mathrm{m} \mathrm{s}^{-1}\right)$, the value that produces the ultrasound image with the highest focus quality at the periosteum. To do so, a twolayer (silicone layer and cutaneous tissue) delay-and-sum algorithm was applied multiple times to the same ultrasound data to reconstruct a set of images of the cutaneous tissue layer obtained with different values of the wave-speed in cutaneous tissue. A rectangular region of interest was manually drawn around the periosteum for calculating the two metrics of focus quality. The wave-speed in cutaneous tissue was chosen as the value that provided maximum focus quality.

The estimates of the radial wave-speed in cortical bone $V_{\text {radial }}$ and the anisotropy form parameter $\beta$ correspond to the values that maximize the focus quality at the endosteum in the transverse and longitudinal images, respectively. A three-layer (silicone layer, cutaneous tissue and cortical bone) delay-and-sum algorithm was applied multiple times on the same ultrasound data to reconstruct a set of images of the cortical bone layer obtained with different values of the searched physical parameter. The value of the radial wave-speed and the anisotropy form parameter were varied within a range determined by prior knowledge found in the literature (Granke et al 2011, Bernard et al 2016); between $3000 \mathrm{~m} \mathrm{~s}^{-1}$ and $4000 \mathrm{~m} \mathrm{~s}^{-1}$ for $V_{\text {radial }}$, and between 0.8 and 2 for $\beta$. For each parameter value, an ultrasound image of the cortical bone layer was reconstructed, and the two metrics of focus quality were computed in a rectangular region of interest that was manually drawn around the endosteum. Figure 5 shows typical variations of the two metrics of focus quality as the value of the searched parameter is varied. Both metrics show a maximum for the same parameter value. $V_{\text {radial }}$ and $\beta$ were chosen as the values that provided maximum focus quality in transverse and longitudinal images, respectively.

\subsection{Measurement of the head-wave velocity for estimating $V_{\text {axial }}$}

In this work, the wave-speed in cortical bone in the direction of the axis of the long bone $V_{\text {axial }}$ was obtained by measuring the velocity of the head-wave. The head-wave is a surface wave propagating along the interface between cutaneous tissue and bone (periosteum). If the ultrasound wavelength in cortical bone (in this work less than $2 \mathrm{~mm}$ ) is smaller than the cortical thickness, the first signal recorded by the array is the head-wave. It travels with the velocity of a compressional wave in cortical bone (Bossy et al 2002, 2004). The measurement of the head-wave velocity is routinely applied by seismologists (Telford et al 1990) and is particularly straightforward if the interface is flat. Therefore an ultrasound acquisition in the longitudinal view (figure $3(\mathrm{~b})$ is ideal since the periosteum can be assumed to be locally flat. In this configuration the head-wave velocity equals $V_{\text {axial }}$, the wave-speed in cortical bone in the axial direction. Panels (a) and (b) in figure 6 show that the relationship between arrival time at the receiver and the source-receiver distance is a linear function. The inverse of the slope of this linear relationship is the propagation velocity of the head-wave. In the two-dimensional Fourier domain (wavenumber versus temporal frequency), the head-wave also appears as a straight line since the relationship between temporal frequency and wavenumber is linear for a non dispersive wave (figures 6(c) and (d)).

Because the periosteum is not parallel to the probe array, a single recording would provide a biased measurement of the head-wave velocity, therefore two sets of recorded signals were analyzed (Telford et al 1990); (1) when the first element of the array is the source and (2) when the last element of the array is the source. The apparentor biased-velocities of the head-wave $V_{1}$ and $V_{2}$ measured from these two configurations were estimated by linear regression in the Fourier domain after applying a denoising filter based on singular value decomposition (SVD) (Minonzio et al 2010). $V_{\text {axial }}$ is then equal to $\left(2 V_{1} V_{2} \cos (\alpha)\right) /\left(V_{1}+V_{2}\right)$ (Telford et al 1990). $\alpha$ is the angle of the interface ( $\alpha=0$ means that the periosteum is parallel to the probe array). The angle $\alpha$ was measured 

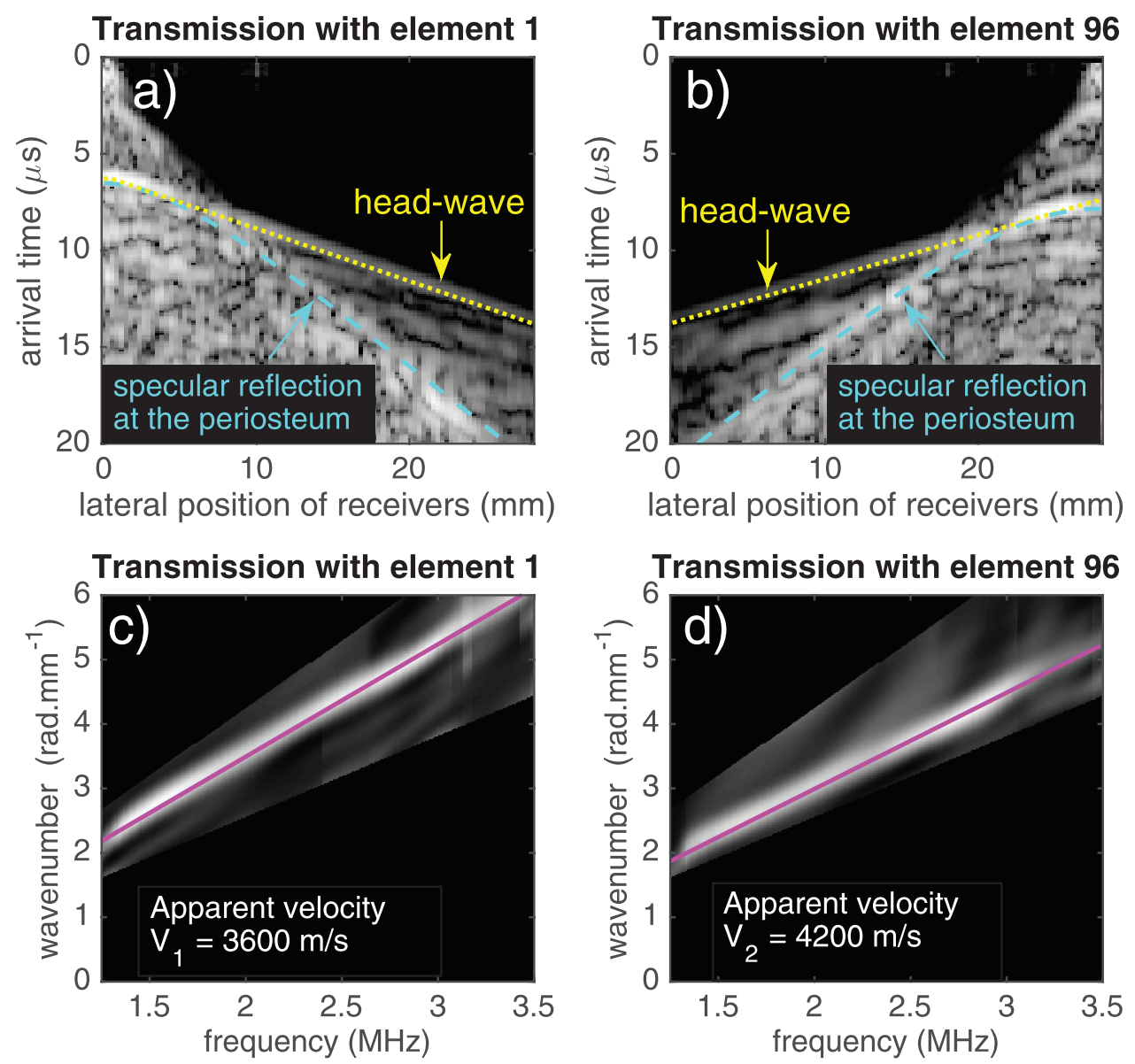

Figure 6. Illustration of the signal processing for the measurement of the velocity of the head-wave at the tibia. Panels (a) and (b) show the log-compressed envelope of the raw signals recorded by the 96 elements when elements 1 and 96 are transmitting, respectively. Panels (c) and (d) show the two-dimensional Fourier transforms of the signals shown in panels (a) and (b) after SVD filtering. A mask mutes the regions corresponding to a wave-speed smaller than $3000 \mathrm{~m} \mathrm{~s}^{-1}$ and larger than $5000 \mathrm{~m} \mathrm{~s}^{-1}$. The observed branch correspond to the head-wave, the result of the linear regression is depicted by a solid line and gives the apparent velocities $V_{1}$ and $V_{2}$. In this measurement, the angle formed by the periosteum and the ultrasound probe was $2^{\circ}$.

in the longitudinal image of the cutaneous tissue layer that was reconstructed after the wave-speed in cutaneous tissue had been estimated.

\subsection{Measurement of the cortical bone thickness at the distal radius with ultrasound and $\mathrm{x}$-ray $\mathrm{CT}$}

The mean cortical thickness was measured in longitudinal ultrasound images where the periosteum and the endosteum can be considered flat. After segmentation of the periosteum and the endosteum using a technique based on Dijkstra's algorithm (Dijkstra 1959, Hong et al 2012) and fitting of the two automatically segmented interfaces with linear functions, the centerline between the periosteum and the endosteum was computed. The cortical thickness was calculated as the mean distance between the periosteum and the endosteum, calculated along a direction that is normal to the centerline. The thickness measured with ultrasonography was compared to that obtained with $\mathrm{x}$-ray CT.

The ground truth cortical thickness of the left radius of the two volunteers was obtained with site-matched high-resolution x-ray peripheral computed tomography (XtremeCT, Scanco Medical, Switzerland; voxel size $82 \mu \mathrm{m})$. All methods were performed in accordance with the relevant guidelines and regulations. The region of interest was scanned with 110 slices starting $75 \mathrm{~mm}$ away from the distal end of the radius and ending $84 \mathrm{~mm}$ away from the distal end of the radius. Figure 7 shows 3D images segmented with Otsu's method (Otsu et al 1979).

The cortical thickness was measured in the anterolateral quadrant where the ultrasound examination was conducted. Five slices in the 3D data set, evenly positioned along the axis of the bone, were chosen. The exact position of the ultrasound probe is unknown, thus the cortical thickness was evaluated by tracing 100 line segments between the periosteum and the endosteum (in the anterolateral quadrant), first with a direction normal to the periosteum and second with a direction normal to the endosteum. The distribution of lengths of these line segments obtained in the five slices provided the mean value and standard deviation (uncertainty) of the cortical thickness. 

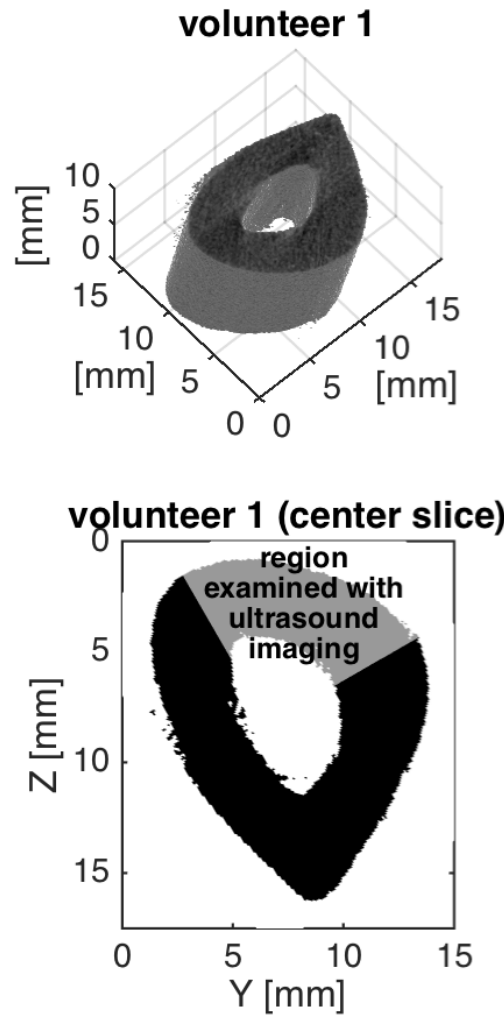
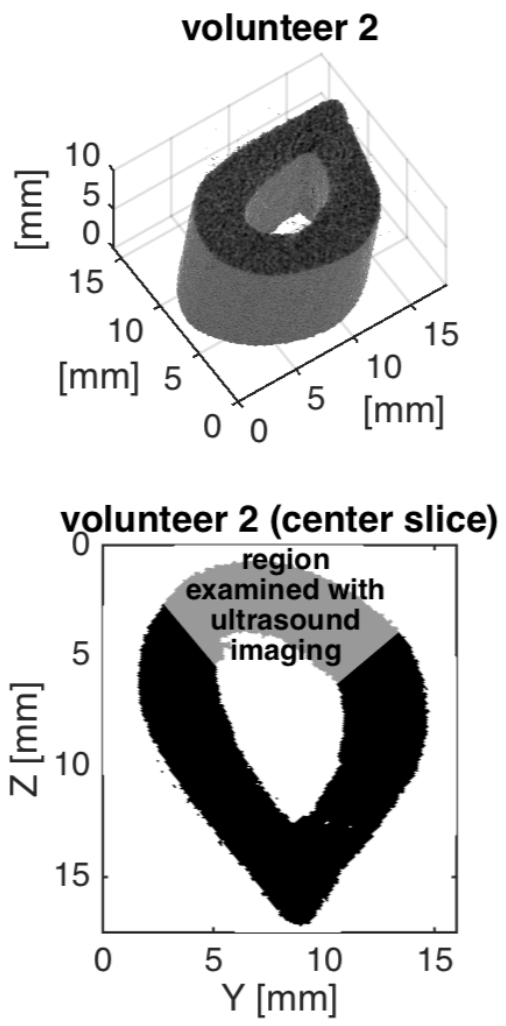

Figure 7. Ground truth anatomy at the distal radius of the two volunteers obtained with high-resolution peripheral x-ray computed tomography. Bottom figures show the center slice, with the anterolateral quadrant examined with ultrasonography highlighted in gray.

\section{Results}

\subsection{In vivo ultrasound images of the radius and tibia}

In figures 8 and 9 we show the result of imaging the radius and tibia using our method. For comparison we also show the images that would have been obtained (using the same data) if only one wave-speed (1540 $\mathrm{m} \mathrm{s}^{-1}$ ) is considered for the entire reconstruction (top panels (a) and (b) in figures 8 and 9), as is done in current clinical scanners. Unlike the images reconstructed with the assumption of uniform wave-speed in the entire region of interest (top panels), the endosteum is clearly visible in the images reconstructed with the method developed in this work (bottom panels). It is important to note that the image reconstruction only stretches until the beginning of the bone marrow, as strong multiple reflections prevent a successful further reconstruction. This issue is further discussed in section 5 . The complete $\mathrm{C}++$ code for image reconstruction of bone is provided in the online supplementary material, together with the set of raw ultrasound data corresponding to panel (d) in figure 8 . The reconstruction of the image shown in figure 8 (panel $(\mathrm{d})$ ) takes a couple of seconds with a state-ofthe-art computer. Computation is parallelized with multi-core processing on CPU. We believe the computational time can be reduced if the code is further optimized and likely with GPU-based parallel programming.

After segmentation of the periosteum and the endosteum, the thickness of the cortical shell at the distal radius was evaluated in the longitudinal images for the two volunteers, following the method described in section 3.4 (figure 10). The cortical thickness was $3.6 \pm 0.1 \mathrm{~mm}$ for volunteer 1 and $3.6 \pm 0.3 \mathrm{~mm}$ for volunteer 2 . These values agree with the thickness measured with high-resolution peripheral x-ray computed tomography (table 1).

The thickness of the cortical shell measured at the tibia (mid-diaphysis) with ultrasonography was $4.9 \pm 0.7 \mathrm{~mm}$ for volunteer 1 and $4.2 \pm 0.3 \mathrm{~mm}$ for volunteer 2 . We were not able to validate these numbers with the $\mathrm{x}$-ray CT images because the high-resolution scanner could not reach the mid-diaphysis of the tibia.

\subsection{Compressional wave-speed and anisotropy in cortical bone}

The wave-speeds measured in cutaneous tissue and cortical bone, and the anisotropy form parameter in cortical bone are summarized in table 2 . The mean compressional wave-speed in cutaneous tissue was calculated by averaging the 10 values obtained in the transverse and longitudinal views. The values are in good agreement with literature (Granke et al 2011, Bernard et al 2016, Moran et al 1995). We found that the axial compressional wavespeed $V_{\text {axial }}$ at the radius was larger than that measured at the tibia, which is also in agreement with previous studies (Njeh et al 2001,Zadik et al 2003, Olszynski et al2013). Interestingly, our method provides a measurement 

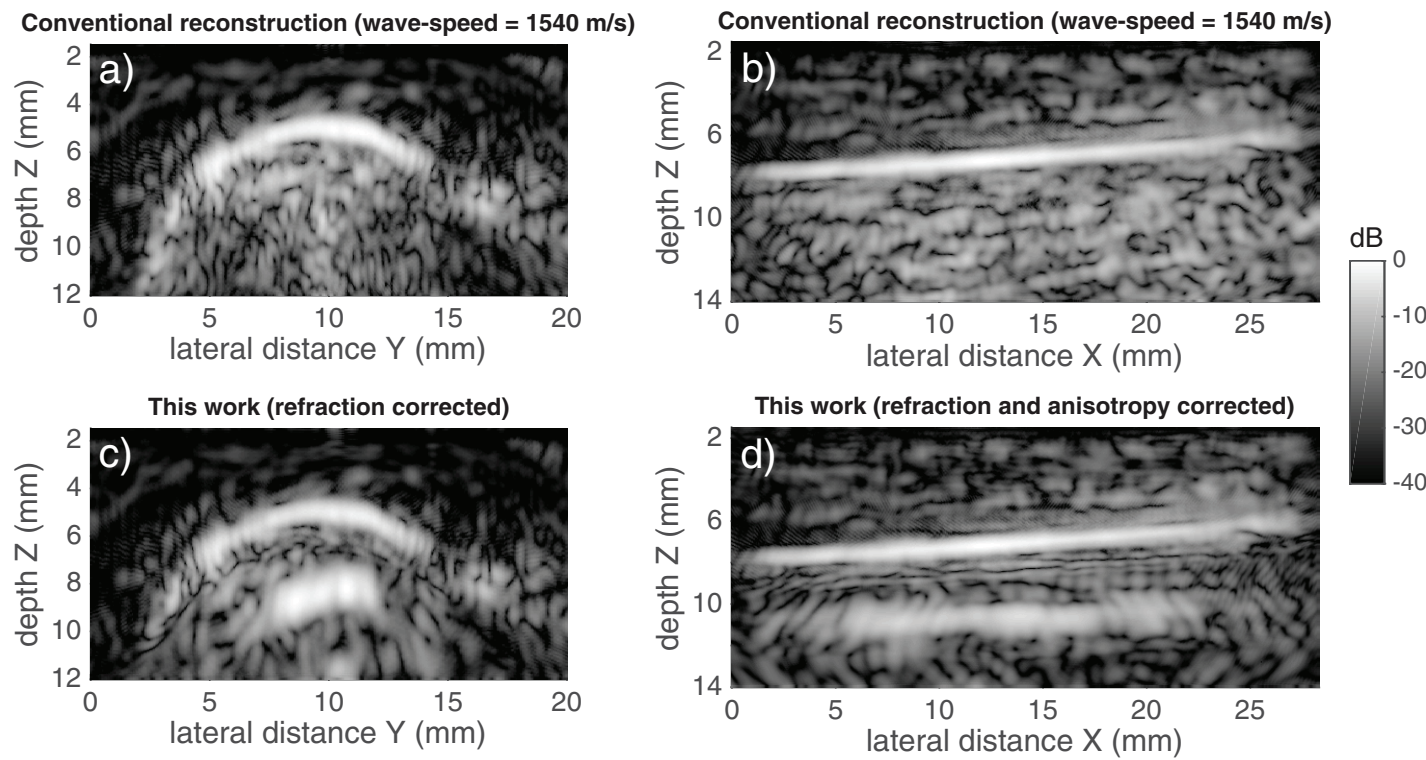

Figure 8. Transverse (panels (a) and (c)) and longitudinal (panels (b) and (d)) ultrasound images of the radius. Top panels ((a) and (b)) show the images obtained with conventional image reconstruction that assumes a uniform medium with a wave-speed of $1540 \mathrm{~m} \mathrm{~s}^{-1}$. Bottom panels ((c) and (d)) show the images reconstructed with the method described in this work that considers refraction and anisotropy in cortical bone. Unlike in panels (a) and (b), the endosteum (inner interface between cortical bone and marrow) is clearly visible in panels (c) and (d). The images are normalized to the maximum pixel value, log-compressed and displayed with a dynamic range of $40 \mathrm{~dB}$.
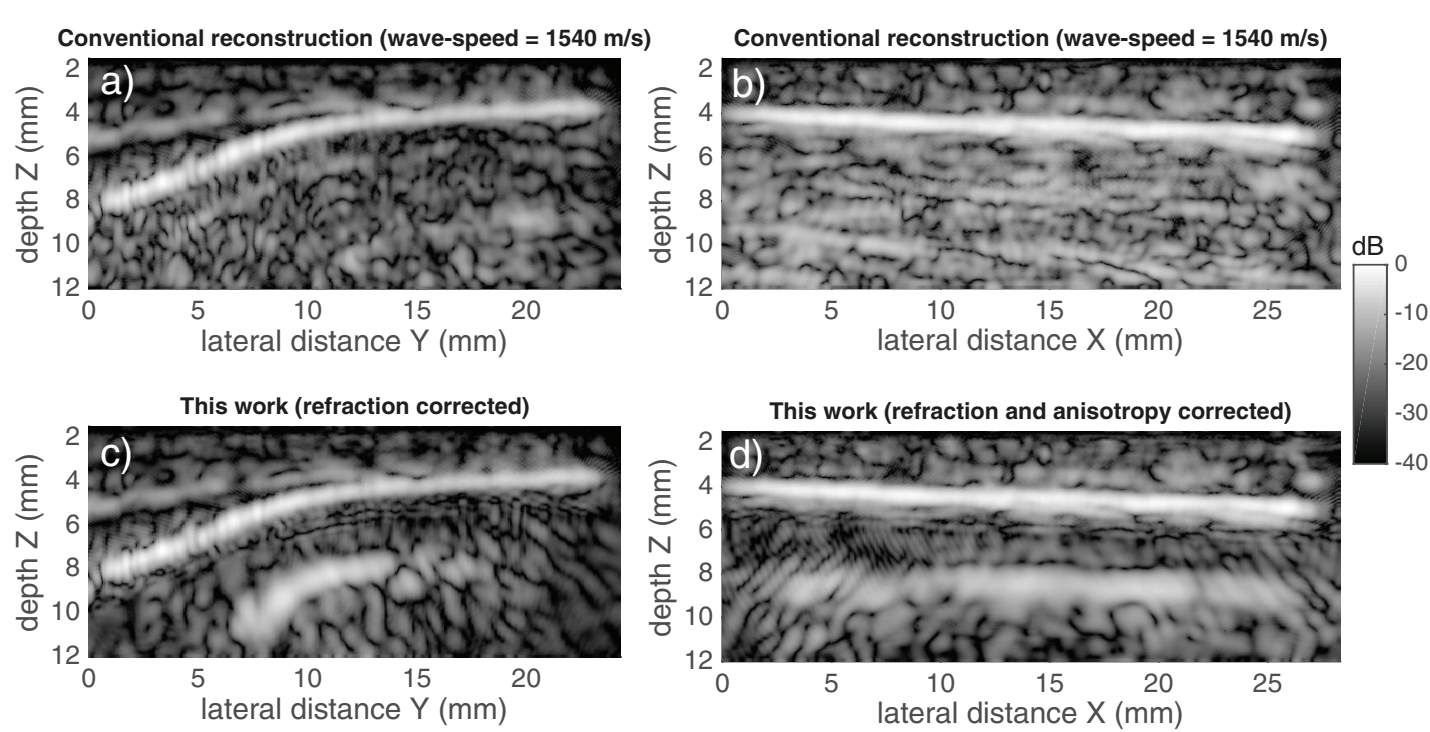

Figure 9. Transverse (panels (a) and (c)) and longitudinal (panels (b) and (d)) ultrasound images of the tibia. Top panels ((a) and (b) show the images obtained with conventional image reconstruction that assumes a uniform medium with a wave-speed of $1540 \mathrm{~m} \mathrm{~s}^{-1}$. Bottom panels ((c) and (d)) show the images reconstructed with the method described in this work that considers refraction and anisotropy in cortical bone. Unlike in panels (a) and (b), the endosteum (inner interface between cortical bone and marrow) is clearly visible in panels (c) and (d). The images are normalized to the maximum pixel value, log-compressed and displayed with a dynamic range of $40 \mathrm{~dB}$.

of the magnitude and form of the elastic anisotropy in cortical bone in vivo. Figure 11 shows the dependence of the group velocity of a compressional wave in cortical bone on the propagation direction, calculated with equation (1) and the estimated values reported in table 2.

\section{Discussion}

\subsection{On the choice of the type of transmit ultrasound beam}

Several types of transmit ultrasound beams have been proposed for ultrasound imaging. In this work we employed a synthetic aperture scheme in which every element in the array subsequently transmits a quasispherical diverging wavefront, followed by a full array capture of the received echo signals. Ultrasound imaging 


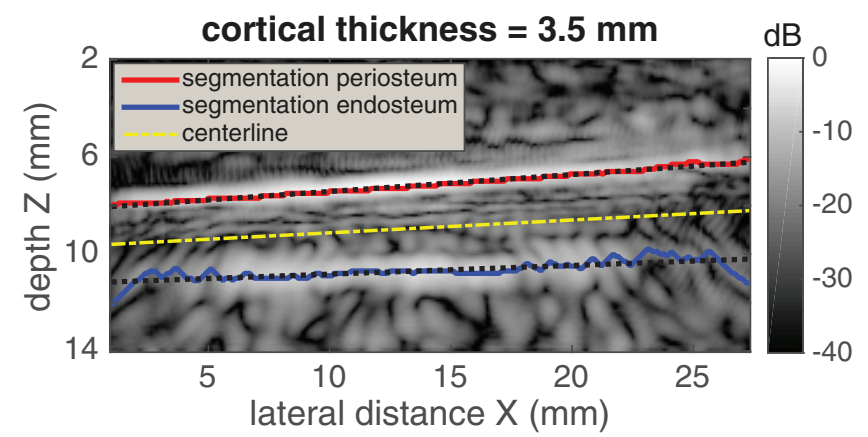

Figure 10. Segmentation of the periosteum and endosteum at the radius. The two segmented interfaces were approximated by linear functions shown as dotted black lines in the figure. The cortical thickness was calculated as the mean distance between the periosteum and the endosteum, calculated along a direction that is normal to the centerline. The cortical thickness for this acquisition is $3.5 \mathrm{~mm}$. Note that the image is the same as that in panel (d) of figure 8.

Table 1. Thickness of cortical bone at the distal radius measured with ultrasound imaging and high-resolution peripheral $\mathrm{x}$-ray computed tomography.

\begin{tabular}{lll}
\hline & $\begin{array}{l}\text { Ultrasound image } \\
\text { Cortical thickness }\end{array}$ & $\begin{array}{l}\text { X-ray image } \\
\text { Cortical thickness }\end{array}$ \\
\hline Volunteer 1 & $3.6 \pm 0.1 \mathrm{~mm}$ & $3.7 \pm 0.2 \mathrm{~mm}$ \\
Volunteer 2 & $3.6 \pm 0.3 \mathrm{~mm}$ & $3.5 \pm 0.3 \mathrm{~mm}$ \\
\hline
\end{tabular}

Table 2. Ultrasound velocities and anisotropy form parameter measured at radius and at tibia (mean and standard deviation are given).

\begin{tabular}{lllll}
\hline & $\begin{array}{l}\text { Cutaneous tissue } \\
\text { wave-speed }\left(\mathrm{m} \mathrm{s}^{-1}\right)\end{array}$ & $\begin{array}{l}\text { Cortical bone radial } \\
\text { wave-speed } V_{\text {radial }}\left(\mathrm{m} \mathrm{s}^{-1}\right)\end{array}$ & $\begin{array}{l}\text { Cortical bone axial } \\
\text { wave-speed } V_{\text {axial }}\left(\mathrm{m} \mathrm{s}^{-1}\right)\end{array}$ & $\begin{array}{l}\text { Cortical bone } \\
\text { anisotropy form parameter } \beta\end{array}$ \\
\hline Radius volunteer 1 & $1680 \pm 30$ & $3240 \pm 140$ & $4000 \pm 70$ & $1.5 \pm 0.2$ \\
Tibia volunteer 1 & $1570 \pm 20$ & $3240 \pm 100$ & $3850 \pm 40$ & $1.4 \pm 0.2$ \\
Radius volunteer 2 & $1610 \pm 30$ & $3240 \pm 100$ & $4020 \pm 60$ & $1.1 \pm 0.2$ \\
Tibia volunteer 2 & $1550 \pm 10$ & $3270 \pm 40$ & $3900 \pm 50$ & $1.1 \pm 0.2$ \\
\hline
\end{tabular}
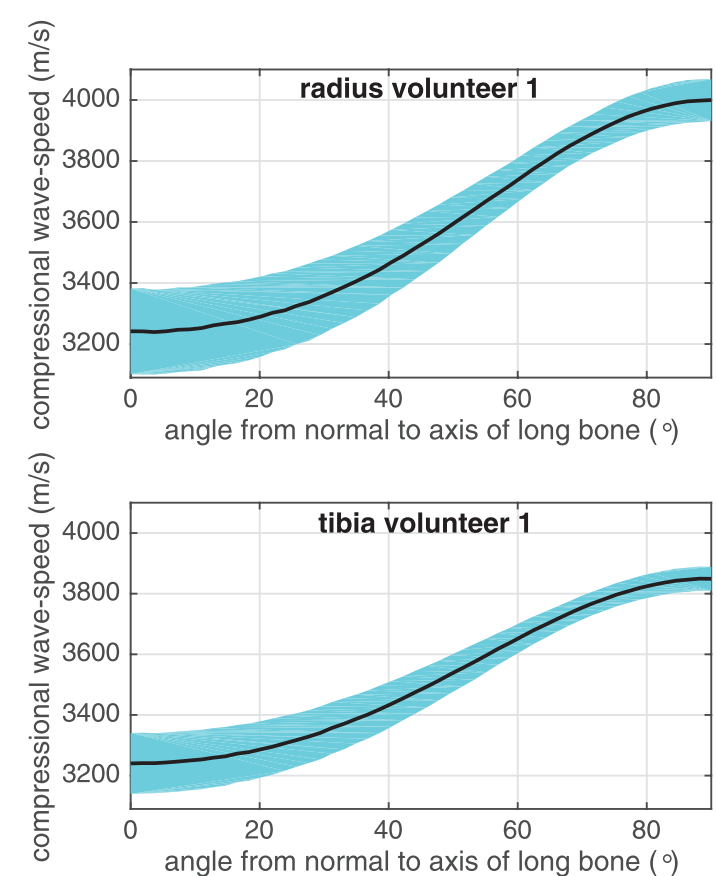
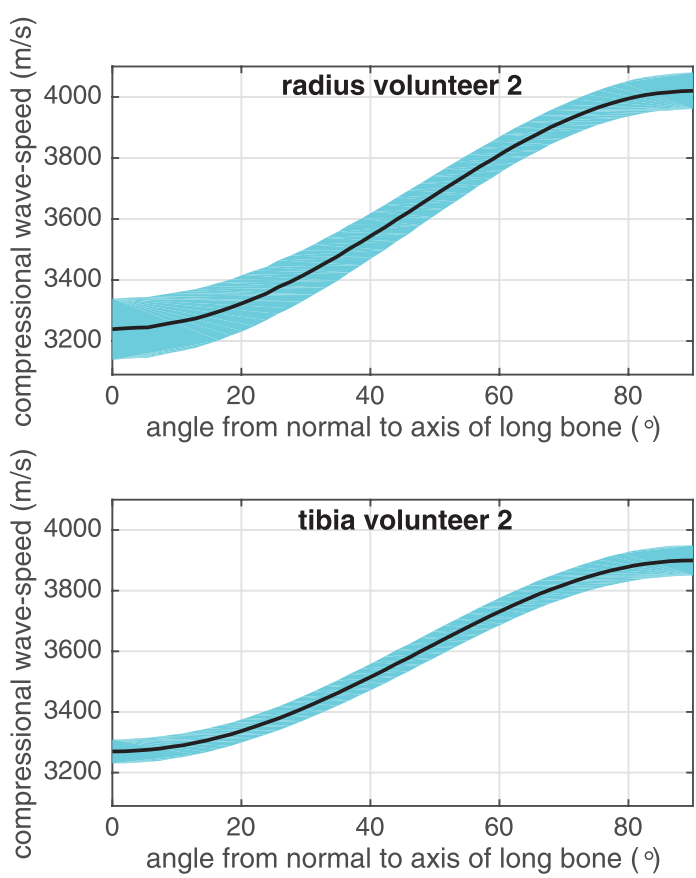

Figure 11. Estimated compressional wave-speed (group velocity) in cortical bone as a function of the propagation direction (ultrasound ray angle), for the two volunteers at the radius and tibia. The solid black line depicts the mean value and the \pm one standard deviation range is shown about the mean value. 
of bone would also be possible with the transmission of plane waves with different steering angles (Montaldo et al 2009). The transmission of plane waves would necessitate the adaptation of the calculation of the transmit travel time in our algorithm. This approach would be beneficial if the signal-to-noise ratio in the recorded pulseecho signals was insufficient. Indeed the full array is activated to generate a plane wave while a synthetic aperture scheme activates one element at a time, and therefore the energy transmitted by a plane wave is higher. The logical basis in conventional ultrasound imaging is to sequentially form straight lines in the image, by selectively insonifying the scatterers that are located on a given image line. The selective insonification of the image lines is performed by transmitting a series of focused narrow ultrasound beams, as many beams as the number of lines in the image (Shung 2015). A transmit focused beam is generated by delaying the excitation of individual elements of the array. The calculation of the transmit delays assumes a medium with a uniform wave-speed. As with image reconstruction, this assumption is acceptable in soft tissues but it does not hold in bone. Because of refraction, a transmit focused beam is distorted when traversing a bone. In particular, the path followed by the transmit focused beam is not a straight line, as it should be. Therefore transmit focused beams as used in conventional ultrasound imaging are not appropriate for imaging bone.

\subsection{Combined characterization of bone anatomy and tissue quality with ultrasound}

Cortical bone thickness and ultrasonic wave-speed were proposed as biomarkers of bone mechanical strength (Njeh et al 2001, Zadik et al 2003, Talmant et al 2009, Chevalley et al 2013, Olszynski et al 2013, Moilanen et al 2013). These two biomarkers have been measured by dedicated ultrasound systems that do not allow for the imaging of bone. These systems use ultrasound probes with separate transmit and receive apertures. One approach consists in measuring the velocity of the first arriving signal arising from guided propagation within the cortical shell of a long bone (Njeh et al 2001, Talmant et al 2009, Kilappa et al 2011). In the frequency range exploited by these systems $(0.1-1 \mathrm{MHz})$, the measured velocity is determined by the compressional wave-speed and the bone thickness (Kilappa et al 2011). Therefore a difference in the absolute value of the velocity, measured on two subjects or measured on the same subject at different times, must be interpreted with caution since compressional wave-speed and bone thickness can vary independently. In another approach, a simple pulse-echo measurement through the cortical shell of a long bone with a single-element ultrasound probe was proposed to estimate the cortical thickness, but the compressional wave-speed in cortical bone was assumed to be known (Wear et al 2003). To overcome the limitations of these methods, Vallet et al Vallet et al (2016) recently applied in vivo a more sophisticated approach, based on the multi-modal analysis of guided ultrasound waves (Lamb wave modes). Solving the inverse problem gives the cortical thickness, the wave-speed and its anisotropy in cortical bone. Ex vivo studies have shown that the elastic constants of cortical bone are essentially determined by the cortical porosity (Granke et al 2011). Capitalizing on these ex vivo measurements, Vallet et al (2016) proposed to reduce the number of unknowns in the inverse problem to two parameters; the cortical thickness and the cortical porosity. Solving this two-dimensional inverse problem remains challenging, in particular because the model neglects the influence of cutaneous tissue.

In this work, we developed a method for measuring in vivo the ultrasonic wave-speed and its anisotropy in the bone cortex and reconstructing an accurate image of the bone cortex. It consists of a sequence of optimization problems with a single unknown parameter. The main assumption is the homogeneity of the cutaneous tissue layer and the cortical bone layer. Our method is the first in vivo approach that provides a direct measurement of the magnitude and form of the elastic anisotropy of cortical bone (figure 11), by applying a model of weak elastic anisotropy (equation (1)) and estimating three parameters $\left(V_{\text {axial }}, V_{\text {radial }}\right.$ and $\left.\beta\right)$. The estimated values of those parameters lead to an estimation of the cortical thickness at the distal radius that agrees with the cortical thickness measured with high-resolution peripheral x-ray computed tomography.

\subsection{Can one neglect the elastic anisotropy of cortical bone tissue for ultrasonography?}

In this work we consider the elastic anisotropy of cortical bone tissue (transverse isotropy) for reconstructing the longitudinal ultrasound images. Indeed the wave-speed in cortical bone tissue is about $20 \%$ higher in the direction parallel to the axis of a long bone than in the direction normal to the axis. Taking into account the elastic anisotropy of cortical bone makes the algorithm of ray tracing for image reconstruction (see section 2) more complicated and increases computational time. Therefore it is reasonable to question the added value of considering elastic anisotropy. If the elasticity of cortical bone tissue is assumed isotropic, then equation (1) is not needed and the wave-speed in cortical bone has a constant value $V_{\text {bone }}$. A first choice for the value of $V_{\text {bone }}$ is $V_{\text {radial }}$ reported in table 2. $V_{\text {radial }}$ is the compressional wave-speed in cortical bone evaluated with transverse images where the assumption of isotropic wave-speed is valid. Figure 12 shows a longitudinal image reconstructed with the anisotropic model (panel (a)) and with an isotropic model taking $V_{\text {bone }}=V_{\text {radial }}$ (panel (b)). Clearly the image quality in panel (b) is inferior to that in panel (a), and the endosteum is difficult to identify in panel (b).

Next, we searched for the optimal value of $V_{\text {bone }}$ that provides the maximum intensity and sharpness (as described in section 3.2) in the longitudinal images of the radius, using an isotropic model in cortical bone. 

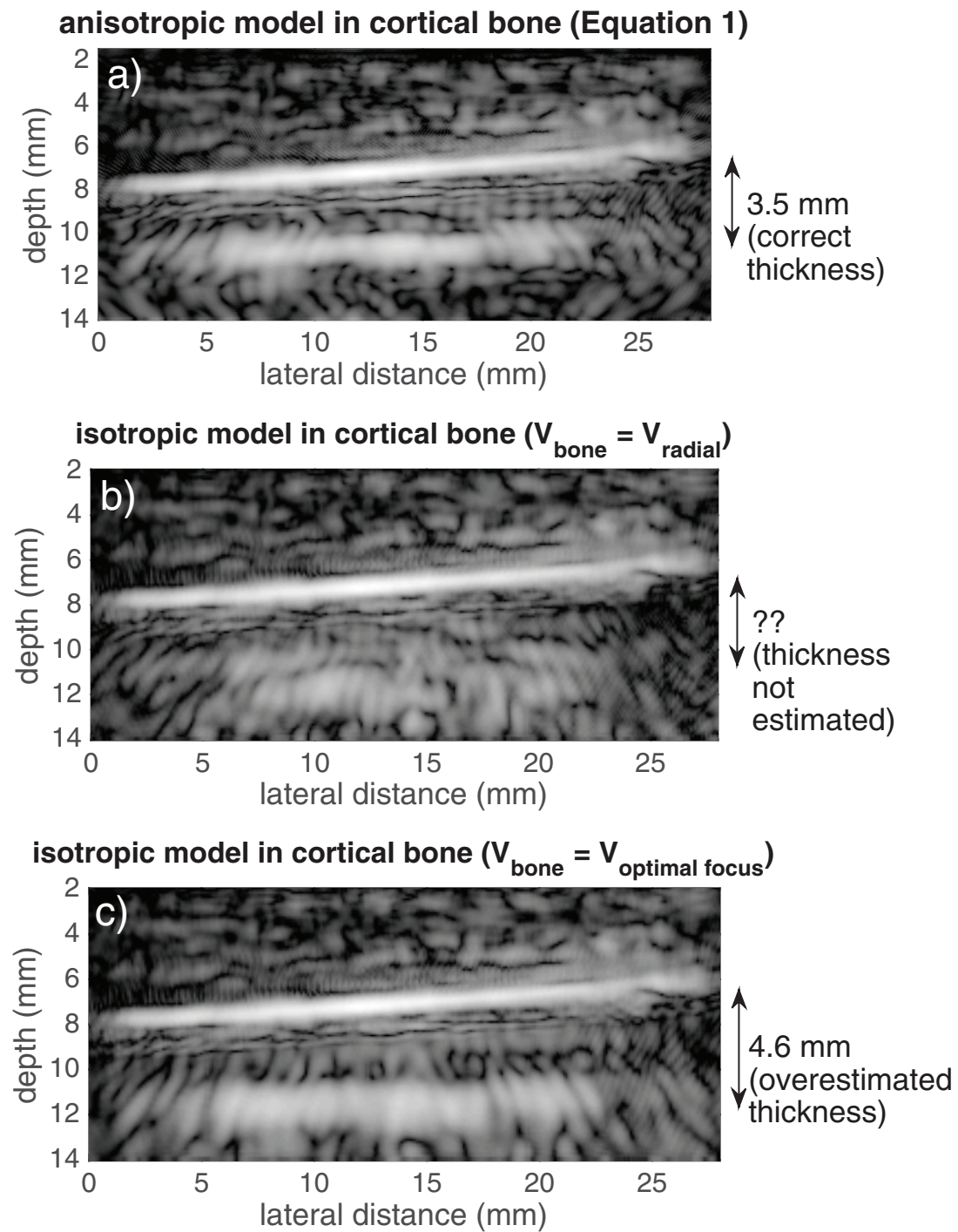

Figure 12. Reconstruction of a longitudinal image of the radius obtained, (a) if the anisotropic model for the wave-speed in cortical bone (equation (1)) and the optimal values (table 2) are used, (b) if an isotropic wave-speed in cortical bone is assumed and taking $V_{\text {radial }}$ in table 2 as the wave-speed in cortical bone, and (c) if an isotropic wave-speed in cortical bone is assumed and the wave-speed in cortical bone is the value that maximizes the focus quality in this longitudinal image $\left(4070 \mathrm{~m} \mathrm{~s}^{-1}\right)$. The image quality in panel (b) is clearly inferior to that in panel (a) (anisotropic model). In panel (c), the image quality is close to that in panel (a) (anisotropic model) but the cortical thickness is overestimated since an optimal isotropic wave-speed of $4070 \mathrm{~m} \mathrm{~s}^{-1}$ is found. Note that the image in panel (a) is the same as that in panel (d) of figure 8 .

The optimal value of $V_{\text {bone }}$ was $3960 \pm 70 \mathrm{~m} \mathrm{~s}^{-1}$ and $4070 \pm 80 \mathrm{~m} \mathrm{~s}^{-1}$ for volunteer 1 and volunteer 2, respectively. Following the methodology described in section 3.4, the cortical thickness at the distal radius was measured in the images. The cortical thickness was $4.5 \pm 0.2 \mathrm{~mm}$ for volunteer 1 and $4.6 \pm 0.3 \mathrm{~mm}$. Thus neglecting elastic anisotropy of cortical bone overestimates the cortical thickness, since the ground truth thickness measured with high-resolution peripheral x-ray computed tomography was $3.7 \pm 0.2 \mathrm{~mm}$ and $3.5 \pm 0.3 \mathrm{~mm}$ for volunteer 1 and volunteer 2, respectively (table 1). Figure 12 shows in panel (c) the ultrasound image reconstructed with $V_{\text {bone }}=4070 \mathrm{~m} \mathrm{~s}^{-1}$, the value that maximizes the focus quality in this longitudinal image, with an isotropic model in cortical bone. Perhaps surprisingly, similar image intensity and image sharpness are obtained at the endosteum in panels (a) and (c). Because the elastic anisotropy of cortical bone is weak, the travel times calculated for image points located on the true endosteum in panel (a) are very close to those calculated for image points located on the erroneous endosteum in panel (c). As a result, the delay-and-sum algorithm produces two images of equivalent quality but only the image in panel (a) is correct because the correct velocity model was used to calculate the travel times.

We therefore conclude that the elastic anisotropy of cortical bone must be taken into account to allow for accurate image reconstruction of the bone cortex in the longitudinal view. 


\subsection{Can the entire cross-section of a bone be imaged?}

We demonstrated in this study the in vivo feasibility of reconstructing images from the first section of long bone. In order to succeed in imaging the entire bone using ultrasound several issues need to be addressed first. Here we discuss two of these: attenuation and multiple reflections.

\subsubsection{Attenuation}

Ideally an ultrasound probe operating at a higher frequency may be used to improve the spatial resolution in the image. However, like for conventional ultrasound imaging, ultrasound attenuation imposes a trade-off between spatial resolution and penetration depth (Shung 2015). A simple one-dimensional calculation of the pressure amplitude decay caused by acoustic impedance mismatch and attenuation was used to estimate the pressure amplitude received by the ultrasound probe after a round-trip through an entire section of bone. We chose a configuration that resembles the mid-diaphysis of a tibia: a $1 \mathrm{~cm}$-thick layer of soft tissue, a $5 \mathrm{~mm}$-thick layer of cortical bone, $1.5 \mathrm{~cm}$-thick layer of marrow, and a second $5 \mathrm{~mm}$-thick layer of cortical bone. We used typical values of frequency-dependent attenuation: $0.5 \mathrm{~dB} / \mathrm{cm} / \mathrm{MHz}$ for cutaneous tissue and marrow, $10 \mathrm{~dB} / \mathrm{cm} / \mathrm{MHz}$ for cortical bone (Zheng et al 2007, Shung 2015). The mass density of cortical bone was $1900 \mathrm{~kg} \mathrm{~m}^{-3}$ and the mass density of cutaneous tissue and marrow was $1000 \mathrm{~kg} \mathrm{~m}^{-3}$. The wave-speed in cutaneous tissue, marrow and cortical bone was $1540 \mathrm{~m} \mathrm{~s}^{-1}, 1410 \mathrm{~m} \mathrm{~s}^{-1}$ and $3250 \mathrm{~m} \mathrm{~s}^{-1}$, respectively. We found that an ultrasound wave with a frequency of $4.5 \mathrm{MHz}$ is attenuated by about $120 \mathrm{~dB}$ when it returns to the ultrasound probe. The receive dynamic range of a state-of-the-art ultrasound system is close to $120 \mathrm{~dB}$ (Shung 2015). Therefore the reflection at the end of the second layer of cortical bone can be recorded if the ultrasound frequency is lower than $4.5 \mathrm{MHz}$. This justifies why an ultrasound probe with a center frequency of $2.5 \mathrm{MHz}$ was chosen in this work. Such a probe together with a state-of-the-art scanner are capable of recording the echoes necessary for the reconstruction of the complete cross-section image of a bone.

\subsubsection{Multiple reflections}

The main reason for not reconstructing the complete cross-section image of the bone is the existence of strong multiple reflections. They are produced by a reflection on the surface of the probe (outer or inner surface of the silicon front layer) and two reflections on bone interfaces (periosteum or endosteum). The travel time of these surface multiple reflections can be close to that of the primary reflection on the second inner surface in bone (endosteum) and the second outer surface in bone (periosteum). As a result, the true anatomical interfaces cannot be easily identified. Ideally these multiple reflections must be removed (or attenuated) in the received raw signals before image reconstruction. Further work is required to implement this pre-processing step.

\subsection{Clinical usefulness of ultrasound imaging of the bone cortex}

Ex vivo studies have shown that cortical bone is an important determinant of the mechanical strength of a bone, because it carries a large amount of the mechanical loading applied to a bone (Bala et al 2015). These findings have been corroborated by clinical studies showing that the measurement of the cortical thickness improves fracture prediction (Chevalley et al 2013, Yang et al 2014). The measurement of the cortical thickness in clinical studies is commonly performed with high-resolution peripheral x-ray computed tomography (Chevalley et al 2013). However this technique uses ionizing radiation and the width of a longitudinal image is limited to $9 \mathrm{~mm}$. High-resolution peripheral $\mathrm{x}$-ray computed tomography is applicable to distal peripheral sites only (distal radius or distal tibia), but there exists ongoing research on the use of clinical $\mathrm{x}$-ray computed tomography and advanced analysis to estimate the cortical thickness at the proximal femur (Treece et al 2012, Humbert et al 2016). On the contrary, ultrasound imaging is a non-ionizing technique and is relatively inexpensive. In this work, we showed that ultrasound imaging provides an accurate measurement of the cortical thickness at the radius. Therefore it could become an alternative to high-resolution peripheral x-ray computed tomography for the measurement of the cortical thickness, in particular for pediatric radiology because the potential risk associated with radiation exposure is higher in children than in adults. Moreover ultrasound imaging is able to produce a longitudinal image with a larger width ( $28 \mathrm{~mm}$ in this work) than high-resolution peripheral x-ray computed tomography $(9 \mathrm{~mm})$. Finally, the measurement of the cortical thickness with ultrasound imaging has the potential to be applied to the central skeleton (hip and spine for instance (Conversano et al 2015, Pisani et al 2016)).

Besides the cortical thickness, material properties such as the elastic properties of cortical bone tissue also play an important role in bone strength (Bala et al 2015). Unlike $\mathrm{x}$-ray, ultrasound senses elasticity of the matter where it travels. The ultrasonic wave-speed is determined by the elasticity and was proposed as a biomarker of the quality of cortical bone tissue (Njeh et al 2001, Zadik et al 2003, Talmant et al 2009, Olszynski et al 2013, Moilanen et al 2013). In this work, we showed that the ultrasonic wave-speed in cortical bone and its anisotropy can be measured in vivo. Once measured, the ultrasonic wave-speed in cortical bone and its anisotropy lead to accurate ultrasound imaging of the bone cortex. 


\section{Conclusions}

This work shows that ultrasound imaging of the cortical shell of a long bone is possible in vivo if refraction and elastic anisotropy of cortical bone are considered. Longitudinal and transverse ultrasound images of the radius and tibia of two healthy volunteers were obtained with a $2.5 \mathrm{MHz}$ linear phased-array transducer. The developed methodology leads to an estimation of the compressional wave-speed and anisotropy in cortical bone. The wave-speed in cutaneous tissue, the wave-speed in cortical bone and the elastic anisotropy in cortical bone were evaluated by searching for optimal focus quality (maximum intensity and sharpness) in the ultrasound image. These optimal values lead to an estimation of the cortical thickness at the distal radius that agrees with the cortical thickness measured with high-resolution peripheral x-ray computed tomography. We also show that the elastic anisotropy of cortical bone must be considered in order to reconstruct accurate longitudinal ultrasound images. Our approach is the first to achieve in vivo a combined characterization of the thickness of the bone cortex, the ultrasonic compressional wave-speed in cortical bone and its anisotropy (magnitude and form).

\section{Acknowledgments}

We thank our colleagues at Lariboisière hospital (Paris), Christine Chappard and Sylvie Fernandez, for helping with the HR-pqCT scans. This work has been funded by Émergence Sorbonne Université (FUIBCA SU-16-REMR-51 grant).

\section{ORCID iDs}

Guillaume Renaud (1) https://orcid.org/0000-0002-6666-1114

\section{References}

Beltrame V, Stramare R, Rebellato N, Angelini F, Frigo A C and Rubaltelli L 2012 Sonographic evaluation of bone fractures: a reliable alternative in clinical practice? Clin. Imaging 36 203-8

Bernard S, Schneider J, Varga P, Laugier P, Raum K and Grimal Q 2016 Elasticity-density and viscoelasticity-density relationships at the tibia mid-diaphysis assessed from resonant ultrasound spectroscopy measurements Biomech Model Mechanobiol. 15 97-109

Bernard S, Monteiller V, Komatitsch D and Lasaygues P 2017 Ultrasonic computed tomography based on full-waveform inversion for bone quantitative imaging Phys. Med. Biol. 627011

Bossy E, Talmant M and Laugier P 2002 Effect of bone cortical thickness on velocity measurements using ultrasonic axial transmission: A 2D simulation study J. Acoust. Soc. Am. 112 297-307

Bossy E, Talmant M, Defontaine M, Patat F and Laugier P 2004 Bidirectional axial transmission can improve accuracy and precision of ultrasonic velocity measurement in cortical bone: a validation on test materials IEEE Trans. Ultrason. Ferroelectr. Freq. Control 51 71-9

Bala Y,Zebaze R and Seeman E 2015 Role of cortical bone in bone fragility Curr. Opin. Rheumatol. 27 406-13

Chevalley T, Bonjour J, van Rietbergen B, Ferrari S and Rizzoli R 2013 Fracture history of healthy premenopausal women is associated with a reduction of cortical microstructural components at the distal radius Bone 55 377-83

Conversano F et al 2015 A novel ultrasound methodology for estimating spine mineral density Ultrasound Med. Biol. 41 281-300

Dijkstra E 1959 A note on two problems in connexion with graphs Numer. Math. 1 269-71

Etgen J, Gray S and Zhang Y 2009 An overview of depth imaging in exploration geophysics Geophysics 74 WCA5-WCA17

Granke M, Grimal Q, Saïed A, Nauleau P, Peyrin F and Laugier P 2011 Change in porosity is the major determinant of the variation of cortical bone elasticity at the millimeter scale in aged women Bone 49 1020-6

Hong D 2012 Medical Image Segmentation Based on Accelerated Dijkstra Algorithm (Berlin: Springer) pp 341-8

Holmes C, Drinkwater B and Wilcox P 2005 Post-processing of the full matrix of ultrasonic transmit-receive array data for non-destructive evaluation NDT \& E Int. 38 701-11

Humbert L, Marangalou J, del Rio Barquero L, van Lenthe G and van Rietbergen B 2016 Technical note: cortical thickness and density estimation from clinical ct using a prior thickness density relationship Med. Phys. 43 1945-54

Jensen J, Nikolov S, Gammelmark K and Pedersen M 2006 Synthetic aperture ultrasound imaging Ultrasonics 44 e5-15

Kilappa V, Moilanen P, Xu L, Nicholson P H F, Timonen J and Cheng S 2011 Low-frequency axial ultrasound velocity correlates with bone mineral density and cortical thickness in the radius and tibia in pre- and postmenopausal women Osteoporos Int. 22 1103-13

Lasaygues P, Ouedraogo E, Lefebvre J-P, Gindre M, Talmant M and Laugier P 2005 Progress towards in vitro quantitative imaging of human femur using compound quantitative ultrasonic tomography Phys. Med. Biol. 50 p 2633

Lasaygues P 2006 Assessing the cortical thickness of long bone shafts in children, using two-dimensional ultrasonic diffraction tomography Ultrasound Med. Biol. 32 1215-27

Li H, Le L H, Sacchi M D and Lou E H M 2013 Ultrasound imaging of long bone fractures and healing with the split-step fourier imaging method Ultrasound Med. Biol. 39 1482-90

Minonzio J, Talmant M and Laugier P 2010 Guided wave phase velocity measurement using multi-emitter and multi-receiver arrays in the axial transmission configuration J. Acoust. Soc. Am. 127 2913-9

Moran C M, Bush N L and Bamber J C 1995 Ultrasonic propagation properties of excised human skin Ultrasound Med. Biol. 21 1177-90

Montaldo G, Tanter M, Bercoff J, Benech N and Fink M 2009 Coherent plane-wave compounding for very high frame rate ultrasonography and transient elastography IEEE Trans. Ultrason. Ferroelectr. Freq. Control 56 489-506

Moilanen P et al 2013 Discrimination of fractures by low-frequency axial transmission ultrasound in postmenopausal females Osteoporosis Int. 24723-30

Nikolov S, Kortbek J and Jensen J 2010 Practical applications of synthetic aperture imaging Proc. IEEE Int. Ultrasonics Symp. pp 350-8 
Njeh C, Saeed I, Grigorian M, Kendler D, Fan B, Shepherd J, McClung M, Drake W and Genant H 2001 Assessment of bone status using speed of sound at multiple anatomical sites Ultrasound Med. Biol. 27 1337-1345

Olszynski W, Brown J, Adachi J, Hanley D, Ioannidis G and Davison K 2013 Multisite quantitative ultrasound for the prediction of fractures over 5 years of follow-up: the canadian multicentre osteoporosis study J. Bone Mineral Res. 28 2027-34

Otsu N 1979 A threshold selection method from gray-level histograms IEEE Trans. Syst. Man Cybern. 962-6

Pisani P, Conversano F, Chiriaco F, Quarta E, Quarta L, Muratore M, Lay-Ekuakille A and Casciaro S 2016 Estimation of femoral neck bone mineral density by ultrasound scanning: preliminary results and feasibility Measurement 94 480-6

Shung K 2015 Diagnostic Ultrasound: Imaging and Blood Flow Measurements 2nd edn (Boca Raton, FL: CRC Press)

Thomsen L 1986 Weak elastic anisotropy Geophysics 51 1954-66

Treeby B, Varslot T, Zhang E, Laufer J and Beard P 2011 Automatic sound speed selection in photoacoustic image reconstruction using an autofocus approach J Biomed. Opt. 16090501

Telford W, Geldart L and Sheriff R 1990 Applied Geophysics 2nd edn (Cambridge: Cambridge University Press) ch 4 (https://doi. org/10.1017/CBO9781139167932)

Talmant M, Kolta S, Roux C, Haguenauer D, Vedel I, Cassou B, Bossy E and Laugier P 2009 In vivo performance evaluation of bi-directional ultrasonic axial transmission for cortical bone assessment Ultrasound Med. Biol. 35 912-9

Treece G, Poole K and Gee A 2012 Imaging the femoral cortex: Thickness, density and mass from clinical ct Med. Image Anal. 16 952-65

Vallet Q, Bochud N, Chappard C, Laugier P and Minonzio J 2016 In vivo characterization of cortical bone using guided waves measured by axial transmission IEEE Trans. Ultrason. Ferroelectr. Freq. Control 63 1361-71

Waltham D 1988 Two-point ray tracing using fermat's principle Geophys. J. Int. 93 575-82

Wear KA 2003 Autocorrelation and cepstral methods for measurement of tibial cortical thickness IEEE Trans. UFFC 50 655-60

Yang L, Udall W J M, McCloskey E V and Eastell R 2014 Distribution of bone density and cortical thickness in the proximal femur and their association with hip fracture in postmenopausal women: a quantitative computed tomography study Osteoporos Int. 25 251-63

Zadik Z, Price D and Diamond G 2003 Pediatric reference curves for multi-site quantitative ultrasound and its modulators Osteoporosis Int. $14857-62$

Zheng R, Le L, Sacchi M, Ta D and Lou E 2007 Spectral ratio method to estimate broadband ultrasound attenuation of cortical bones in vitro using multiple reflections Phys. Med. Biol. 525855

Zheng R, Le L H, Sacchi M D and Lou E 2015 Imaging internal structure of long bones using wave scattering theory Ultrasound Med. Biol. $412955-65$ 